\title{
Development of the Short Time Signals Processing Unit Using an Embedded System
}

\author{
Jozef Hrbček, Emília Bubeníková, Aleš Janota \\ University of Žilina, Faculty of Electrical Engineering and Information Technology, \\ Department of Control and Information Systems, 01026 Žilina, Slovakia \\ \{jozef.hrbcek, emilia.bubenikova, ales.janota\}@fel.uniza.sk
}

\begin{abstract}
This paper deals with the problem of data collection and processing as a part of the Weight-inMotion system. The authors present the concept and technical solution of the short time signals processing unit that addresses the given requirements.
\end{abstract}

Keywords - programmable logic controller; measurement; weight; communications; embedded system

\section{INTRODUCTION}

Dynamic weight measurement (WiM - Weigh in Motion or Weigh-in-Motion) systems are used to get required data on vehicles without breaking traffic flow smoothness. WiM is usually described by various standards [18-20] as "the process of measuring the dynamic tire forces of a moving vehicle and estimating the corresponding tire loads of the static vehicle". These systems work autonomously, independently, and they are able to automatically collect data in real time and send them to the traffic central control system. Nowadays, process control, automatic measurement systems and data processing systems often use the programmable logic controllers (PLCs) instead of personal computers (PCs). The architecture and computation power of PLCs are the same as in the case of PCs, but their realization brings some benefits and is suitable for the intended application. The reason is that PLCs, unlike general PCs, have been designed to be placed in a harsh and unstable environment and their circuitry has been designed to survive in areas of substantial temperatures, electrical noise, humidity, mechanical vibration, various interference, etc. [4], [12]. Last but not least, the reliability features are also worth of mentioning, especially in the case of critical applications when various strategies can be used to analyze reliability [13]. In our project dealing with development of a new WiM system, the technical solution is based on PLCs from B\&R Company. Since one of the authors' intentions was to increase the system reliability, they are operated in redundant connection. In this context, the authors focus on the concept of how data coming from the measurement unit are processed together with consideration of communication principles. A processing unit with the embedded device (based on a microprocessor STM32 and Raspberry Pi) has been designed specifically for the WiM application. In the initial development phase Raspberry Pi has been used to implement industrial Ethernet POWERLINK (EPL) and test its transmissions properties. In future development phases the Raspberry $\mathrm{Pi}$ may be omitted and the communication protocol will be implemented directly into the microprocessor. The paper does not address the measurements unit itself - its physical principle, design, construction features, etc. Some details about its researched principles are available in [3] and [5], the problems resulting from usage of fiber optics in WiM applications are discussed e.g. in [6] and [14].

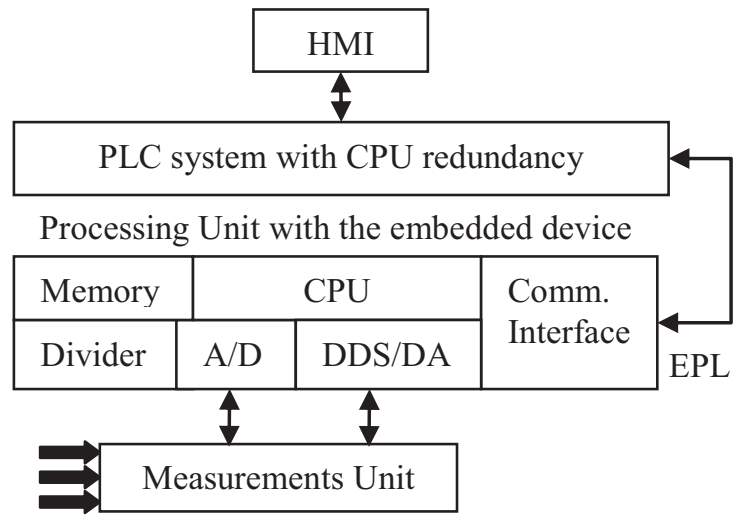

Figure 1. The measurement system concept

\section{THE MAIN CONCEPT}

The main concept is depicted in Figure 1. Based on the chosen physical measurement principle, the design solution must take into account the particularities of operation in external (outdoor) conditions. The processing unit separates the detected signal and transmits it through the communication interface. It uses a specially designed algorithm to calculate the applied load and sends the appropriate signal to the PC through the PLC system. The system may also be equipped with surveillance cameras. More information about the digital image processing can be found in [10] and [17]. Cooperation between vision systems and automation solutions based on the industrial communication protocol opens up new perspectives. The collaboration between Cognex and B\&R enables to implement cameras for the visual surveillance-based solution. The PLC system with CPU redundancy provides a layer between the processing unit and superior level (HMI). This concept realizes the reliable operation of the device under operating conditions. The required operating temperatures are from $-25^{\circ} \mathrm{C}$ to $60{ }^{\circ} \mathrm{C}$

\section{A. Measurement unit}

Although the attention is not drawn here to the physical principle of the used measurement unit, a reader may find literature review on the topic in [15] or survey of known principles in [16]. In this study case totally three principles are the subject of research, 
based on detection of the modulated optical signal [3]. The paper focuses on the rest of the measurement chain whose main parts are the processing unit and the PLC system.

\section{B. Processing unit requirements}

The requirements specification of the processing system results from the initial requirements defined in [3], based on recent findings and practical experience. These requirements are met by the STMicroelectronics microcontroller from the ARM Cortex-M3 32-bit STM32 family with a maximum clock frequency $120 \mathrm{MHz}$. Figure 2 illustrates a simplified block diagram of the processing unit.

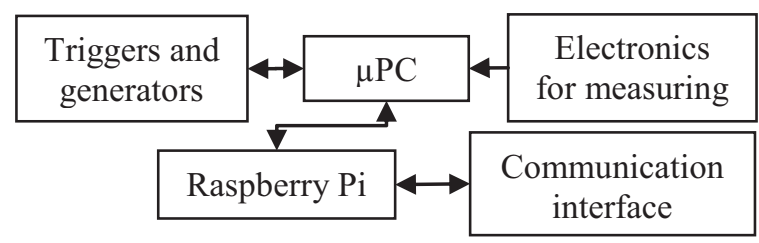

Figure 2. Simplified block diagram of the Processing Unit with the embedded device

\section{PLC system with CPU redundancy}

Modular PLCs consist of modules that can be slotted together to build up a whole system. The basic modules contain a power supply, central processing unit (CPU), communications modules and the input and output modules. It is possible to expand the system by an amount of input and output terminals. Some PLCs use the open source communication networks and allow us to connect the embedded devices to this modular system. We have chosen B\&R technology since it prefers the open communication protocol with high speed (the communication cycle less than $500 \mu \mathrm{s}$ ) and real-time deterministic approach. Moreover, it supports the local and remote visualizations, web server, FTP server and HTTP server for communication with a cloud and the third party devices connection without the need of any additional modules. Usage of the B\&R control system with developed processing unit guarantees the successful solution.

\section{COMMUNICATION PRINCIPLES}

The deterministic, reliable and fast communication bus is the important requirement. The measured data can be sent in packets because the industrial communication networks are not fast enough. Ethernet POWERLINK (EPL) is the open source real-time Ethernet protocol adopting the CANopen object dictionary concept and its communication mechanisms that provide features like interoperability, flexibility and configurability. The lower layers of the EPL software are specifically designed to follow the EPL cycle schedule and to guarantee fast response times for real-time events on the bus. For comparison, the shortest communication cycle time for EPL is $100 \mu \mathrm{s}$ comparing to Profinet's support of $1000 \mu \mathrm{s}$. Embedded systems can be connected as a node of EPL bus by adding the XDD (XML device description) file from the openPOWERLINK source code stack to the programming tool - Automation Studio [11].

\section{INCREASING THE SYSTEM RELIABILITY}

A combination of controller and network redundancy maximizes protection against failures across the entire automation system [2], [7], [8] and [9]. The redundancy of the system serves to restore it after failure. The reserve parts of the system could be omitted if other parts of the system work correctly. Redundancy can be categorized into the following different groups: usage, functionality and fabrication [1]. Our concept of the redundant control system guarantees maximum availability for the entire system. It uses warm redundancy according to the Figure 3. It is necessary to perform a failure mode analysis for the parts of critical system. The system has many potential ways of failing, it has multiple failure modes. The designed system can still function in case of hardware or its software failure (PLC), power failure and EPL network failure. Master redundancy with EPL synchronizes data with microsecond accuracy and can be switched over in a matter of milliseconds. This functionality is integrated into the PLC with real time operating system and is easy to use. The interface PCI_5LS182.6-1 provides the configurable ring redundancy for EPL.

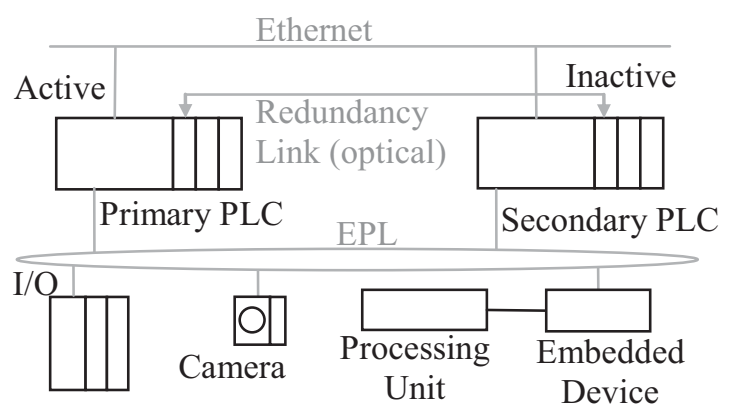

Figure 3. The realised system with CPU redundancy

\section{Processing UnIT}

This processing unit design is divided into two parts: (1) According to the communication interface with other parts of the system; (2) According to the processing data (need a lot of ADC channels and fast sampling, DA converters, trigger and sine generators). For these reasons, we decided to use the Raspberry Pi and STM32F205 microcontroller, which contains everything needed. The microcontroller provides the following measurement functions: sensing the analog values by the internal $\mathrm{AD}$ converters with sampling frequency $2 \mathrm{MHz}$ (signal to noise ratio is $74 \mathrm{~dB}$ ); generating the excitation signals with internal DA converters; generating the variable time base for measurement; communication with DDS generators and external DA converters; whole measurement process.

\section{A. Circuits for measuring the static and dynamic characteristics in the time domain}

Analog values are measured using the internal AD converters. The circuits are realized as voltage dividers with a constant dividing ratio. The reduced signal is then connected into the input buffer for the AD converter that separates the measured system from the processing unit. This solution ensures that the systems 
do not affect each other and the AD converter does not suffer from a lack of current to charge the internal capacities. The voltage dividers are composed of resistors (in the order of $\mathrm{M} \Omega$ ) as well as the input resistance of the operational amplifier used for the system separation, which is approximately $10 \mathrm{M} \Omega$.

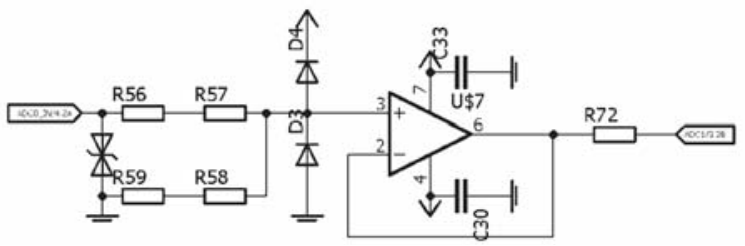

Figure 4. Wiring diagram of the analog input with voltage tracker for saturation of the input channel

The resistors R56 (500 k $\Omega)$, R57 (500 k $\Omega)$, R85 $(7,5 \mathrm{k} \Omega)$ and $\mathrm{R} 59(249 \mathrm{k} \Omega)$ realize the voltage divider. The input is protected by a bi-directional transil with a breakdown voltage of $12 \mathrm{~V}$ and a limitation of the input voltage to the voltage tracker is ensured by the diodes D3 and D4. The high speed operational amplifier LM7171 implements voltage tracker or the current buffer that contains an internal output current limitation and is resistant to ground short-circuit. The $\mathrm{AD}$ converter is a part of the microcontroller.

\section{B. Measurement in the frequency domain}

The device is also ready for use of different measurement principles and therefore contains the Direct Digital Synthesizer (DDS) AD9851 and the phase shift measurement units. The purpose of the circuit with DDS is generating a sinusoidal excitation signal with adjustable output frequency, using the 32bit words, in the range of $42 \mathrm{mHz} \div 180 \mathrm{MHz}$ (in the real system up to $90 \mathrm{MHz}$ ) for the measurement system and process the signals returning from the measured system. We inserted an elliptical reconstruction filter with a $50 \mathrm{MHz}$ frequency band at the output of the integrated DDS generator to remove all higher harmonic components.

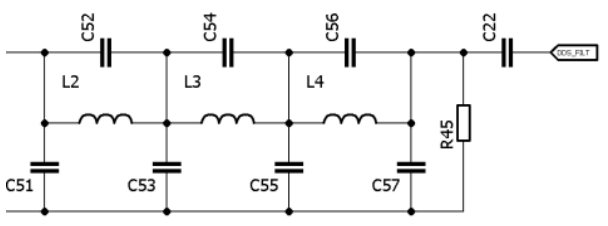

Figure 5. Wiring diagram of the reconstruction filter

The output amplitude is set by the voltagecontrolled amplifier VCA824.

The system operates on the principle similar to a quadrature demodulation where the output signal from the measuring system is connected to the two multipliers in which it is multiplied by the reference signal (or the signal entering the measuring system) and the signal, which is phase-shifted, by $90^{\circ}$ to the reference signal. The outputs from the multipliers are then connected to the low pass filters, where the alternating signal components are eliminated and the resulting unidirectional signal component is passed to the $\mathrm{AD}$ converters. The low pass filter is deactivated in the case of lower frequencies up to $10 \mathrm{kHz}$. The input signal is connected directly to the $\mathrm{AD}$ converter, where it is averaged in the microcontroller to determine its DC component. The obtained values represent $Q$ and $I$ components $(Q$ and $I$ is called "rectangular" or "Cartesian" form, where $I$ is the current momentary amplitude of the signal, $Q$ is the momentary amplitude of the signal phase shifted) from which we calculate the amplitude of the measured signal as follows:

$$
A=\sqrt{Q^{2}+I^{2}}
$$

and the phase shift between the reference signal and the signal from the measured system is expressed as:

$$
\varphi=\tan ^{-1}\left(\frac{Q}{I}\right)\left[{ }^{\circ}\right]
$$

The DDS generator generates the excitation signal (or the reference signal).

\section{Calibration}

Using of a calibrated measuring device (oscilloscope) we performed a measurement of the total attenuation of the processing unit and its phase shift. We have connected the generator output to the processing unit input by a coaxial cable and we performed measurements in the frequency range from $10 \mathrm{~Hz}$ to $30 \mathrm{MHz}$. Each of the measuring network components may have its own attenuation and phase shift at a given frequency, so we have decided to create a software self-calibration that should effectively minimize measurement errors. Using of the test program, the amplitude of the output sinusoidal signal is gradually increased until the measured signal reached the amplitude $1 \mathrm{~V}$. The program saves the value of the set output voltage where this value is used as a calibration constant for a given frequency. The $Q$ and $I$ components of the signal are saved at the same time, from which the phase shift is calculated. This is used as calibration constants for phase shift at a given frequency. The black line shows the calibrated phase shift. On the chart below (Figure 6) we can see the measured attenuation of the device before (dashed grey line) and after calibration (solid grey line).

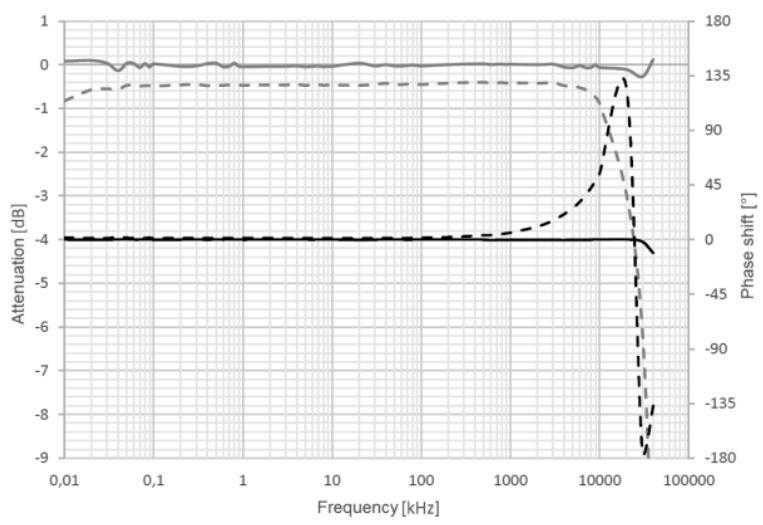

Figure 6. Measured attenuation and phase shift before and after calibration

\section{Additional functionalities}

Furthermore, the processing unit includes three precision peak detector realized by a pair of 
operational amplifiers ADA8417, diode BAT54 and $\mathrm{RC}$ element with the adjustable time constant. The circuit functionality was verified by simulation in the program LTSpice IV. Simulated circuits meet all the functional requirements that have been set.

\section{E. The processing unit accuracy verification}

The last step was to verify the accuracy of the time base generated by the timer of the microcontroller. This is an important parameter for measuring dynamic characteristics in the time domain. We used an indirect method to measure the transition characteristic of the $\mathrm{RC}$ filter using the oscilloscope Rigol DS1054Z and realized measuring device for verification. Both measurements were identical. The real measured signal from measurement system is shown in Figure 7.

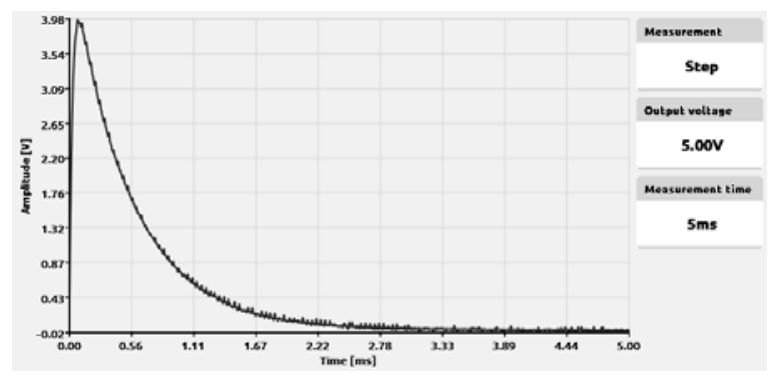

Figure 7. Measured signal displayed on local visualization panel

The processing unit uses the Raspberry Pi with the operating system Raspbian Jessie kernel 4.14 with implemented RT-PREEMPT. Implemented EPL stack version 2.7.1 realizes deterministic communication with the redundant control system. The communication cycle time is now $2000 \mu \mathrm{s}$. The measurement is performed with a period of $0.5 \mu \mathrm{s}$ (for three 12-bit ADC channels) and sent the data in the packet every communication cycle. The available size of windows time is selectable from 1 to $5 \mathrm{~ms}$.

\section{CONCLUSION}

Proper operation of the processing unit has been done by setting of the adjustable parameters such as input and output offsets for the voltage-controlled amplifier, the output amplitude of DDS generators and voltage for the offset of analog multipliers using the test program. The individual ranges of the analog inputs and outputs have been tested. All measured values have been recorded for the calibration purposes. The microcontroller program is written in programming language $\mathrm{C}$ because the available libraries for the microcontroller are also written in the C language. The STM32CubeMX tool has been used for the initial configuration of the microcontroller. Finally, we can evaluate the processing unit as successfully developed with all designed functionality, including the connection with the control and visualization part of the system. This solution fulfills all requirements specified in the main concept. The developed device is usable for the WiM systems.

\section{ACKNOWLEDGEMENT}

The paper has been written with the support of the Slovak Research and Development Agency within the applied research project APVV-15-0441 "OPTIWIM -
Measurement system with optical sensor for WiM systems".

\section{REFERENCES}

[1] J. Hrbček, V. Šimák, A. Janota and R. Pirník, "Tunnel central control system enhanced with modern control approaches," In: Archives of Transport System Telematics, Vol. 7, Issue 3, ISSN 1899-8208, pp. 3-7, September 2014.

[2] B\&R, „Redundancy systems documentation,“ available at: https://www.br-automation.com/en/technologies/redundancy/.

[3] A. Janota, V. Šimák, and J. Hrbček, "Development of a Weight-in-Motion measurement system with an optical sensor," in 17th International Conference on Transport Systems Telematics, Poland, pp. 234-247, ISSN 1865-0929, September 2017.

[4] IEC 61131, "Programmable controllers (PLC)," International standard, part 2: Equipment requirements and tests, 2008.

[5] A. Janota, D. Nemec, M. Hruboš and R. Pirník, "KnowledgeBased Approach to Selection of Weight-in-Motion Equipment," in Communications in Computer and Information Science, vol. 640, Springer, pp. 1-12, 2016.

[6] A. Batenko et al., "Problems of Fibre Optic Sensor Application in Weight-In-Motion (WIM) Systems," in 11th International Conference RelStat'11, pp. 311-316, 2011.

[7] J. Ždánsky and K. Rástočný, "Influence of Redundancy on Safety Integrity of SRCS with Safety PLC," Proceedings of the 10th international conference ELEKTRO 2014, ISBN 978-1-4799-3720-2, Rajecké Teplice, pp. 508-512, May 2014.

[8] K. Rástočný, L. Pekár and J. Ždánsky, "Safety of signalling systems - opinions and reality," Proceedings of the 13th International Conference TST 2013, Ustroń, Poland, Springer, ISBN 978-3-642-41646-0, p. 155-162, October 2013.

[9] K. Rástočný, M. Franeková, P. Holečko and I. Zolotová, "Modelling of hazards effect on safety integrity of open transmission systems," in Computing and Informatics," ISSN 1335-9150, vol. 35, no. 2, p. 470-496, 2016.

[10] J. Halgaš and R. Pirník, "Monitoring of parking lot traffic using a video detection," in Acta Technica Corviniensis, vol. 8, no. 3, ISSN 2067-3809, pp. 17-20, September 2015.

[11] The Ethernet POWERLINK Standardization Group, "An Open Source POWERLINK protocol stack," available at: http://openpowerlink.sourceforge.net/web/, 2019.

[12] C. T. Jones, "Programmable Logic Controllers. The Complete Guide to the Technology," $1^{\text {st }}$ ed., Patrick Turner Publishing Company, 1996.

[13] H. Zhang, Y. Jiang, W. N. N. Hung, G. Yang, M. Gu and J. Sun, "New strategies for reliability analysis of Programmable Logic Controllers. Mathematical and Computer Modelling," vol. 55, issues 7-8, pp. 1916-1931, 2012.

[14] A. Grakovski, A. Pilipovec, I. Kabashkin and E. Petersons, "Weight-in-Motion estimation based on reconstruction of tyre footprint's geometry by group of fibre optic sensors," Transport and Telecommunications, vol. 15, no. 2, pp. 97110, 2014

[15] LTBP Program's Literature „Review on Weight-in-Motion Systems, “ Report No. FHWA-HRT-16-024, June 2016.

[16] Weigh-in-Motion Pocket Guide, Part 1. „WiM Technology, Data Acquisition, and Procurement Guide," FHWA-PL-18015, June 2018.

[17] J.A.B. Madrigal et al., ,Evaluation of suitability of a microprocessing unit of motion analysis for upper limb tracking," in Medical Engineering and Physics, ISSN 1350-4533, vol. 38 , iss. 8, p. 793-800, January 2016.

[18] ASTM Standard E1318-09, "Standard Specification for Highway Weigh-in-Motion (WIM) Systems with User Requirements and Test Methods," West Conshohocken, PA: ASTM International, West Conshohocken, PA, 2009.

[19] European Cooperation in Science and Technology (COST), "Weigh-in-Motion of Road Vehicles," Action 323, ed. B. Jacob at al., 538 p., Paris, 2002.

[20] NMi International WIM Standard, "Specification and Test Procedures for Weigh-in-Motion Systems," NMi Certin, Dordrecht, Netherlands, 2016. 